\title{
DIABETES
}

\section{Epigenetic changes lead to impaired wound healing in patients with T2DM}

Patients with type 2 diabetes mellitus (T2DM) have impaired wound healing owing to the dominance of the proinflammatory M1 macrophage phenotype. New research reveals that, under diabetic conditions, lysine-specific demethylase 6B (also known as JMJD3) demethylates the lysine 27 , histone 3 (H3K27Me3) mark that supresses IL-12 expression, which results in increased IL-12 production and polarization of macrophages to an M1 phenotype.

"Macrophages isolated from patients with T2DM produce excess proinflammatory mediators; however, the molecular mechanisms that programme and sustain these phenotypes were unclear," explains lead author Katherine Gallagher.

Gallagher's team showed that bonemarrow-derived (BMD) stem or progenitor cells and macrophages from glucose intolerant, obese mice had significantly reduced H3K27 methylation in the IL-12 promoter region. In the same cells, and in BMD cells from patients with T2DM, significantly increased JMJD3 and ILI2 gene expression were also apparent. Inhibition of JMJD3 resulted in a significant reduction in IL12 expression in mouse BMD cells. "The $\mathrm{H} 3 \mathrm{~K} 27 \mathrm{Me} 3$ epigenetic mark is passed on to more-differentiated recruited macrophages found in the wound tissue. Thus, peripheral tissue is 'preprogrammed' towards chronic inflammation," Gallagher explains. This finding suggests the H3K27Me3 mark might set a 'metabolic memory' in chronic wound inflammation and other inflammatory diabetic complications. "These mechanisms may provide a novel explanation for the altered clinical phenotypes associated with the pathology of diabetic wounds," Gallagher concludes.

Peter Sidaway

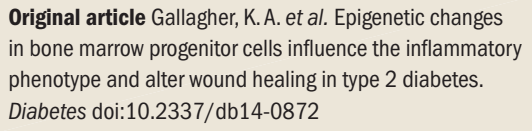

